\title{
Une maladie métabolique à l'origine de la découverte des transporteurs ABCG5 et ABCG8 modulant l'absorption intestinale du cholestérol
}

L'étude des maladies métaboliques héréditaires a permis l'identification et la caractérisation de la fonction de certains gènes. Exemple récent, la maladie de Tangier, de transmission autosomique récessive, qui se caractérise par des taux plasmatiques de HDL très bas et par une incapacité des cellules des sujets atteints à excréter le cholestérol vers l'apolipoprotéine $A-I$. Son étude est à l'origine de la caractérisation du rôle physiologique de ABCA1 (ATP binding cassette A1), une protéine assurant le transport du cholestérol hors des cellules ([1] et $\mathrm{m} / \mathrm{s} 2000, n^{\circ} 3, p .421$ ). C'est encore dans le domaine du métabolisme des lipides qu'une autre maladie métabolique rare, la sitostérolémie, vient de permettre la découverte de deux autres transporteurs, ABCG5 et $A B C G 8$, impliqués dans le contrôle de l'absorption intestinale du cholestérol.

\section{La sitostérolémie: \\ un désordre familial \\ du métabolisme des stérols}

Les deux premiers cas de sitostérolémie ont été décrits en 1973 chez deux sœurs présentant des xanthomes souscutanés dès l'enfance liés à des concentrations plasmatiques en stérols d'origine végétale (sitostérol, campestérol et stigmastérol) anormalement élevées [2]. Cependant, contrairement aux sujets atteints d'hypercholestérolémie familiale (déficit en récepteur des LDL), les concentrations plasmatiques en stérols diminuent ici fortement après un régime alimentaire sans stérols. Cette observation a permis à différents groupes d'envisager que la sitostérolémie pouvait être due à une anomalie de l'absorption intestinale des stérols. Effectivement, on observe $\mathrm{m} / \mathrm{s} \mathrm{n}^{\circ} 6-7$, vol. 17, juin-juillet 2001 chez ces patients, une cinquantaine de cas reportés à ce jour, une absorption intestinale de sitostérol qui peut atteindre 15 à $63 \%$ de la quantité ingérée, alors qu'elle en représente moins de $5 \%$ chez les individus normaux. Seule une petite partie des stérols végétaux ingérés par ces patients est excrétée dans la bile, et le reste s'accumule dans les tissus périphériques à l'exception notable du cerveau. Ces anomalies sont souvent associées à une augmentation de

l'absorption intestinale et de la concentration plasmatique en cholestérol d'origine animale, ce qui peut provoquer l'apparition d'accidents cardiovasculaires, souvent dès un très jeune âge [2]. La sitostérolémie est donc caractérisée par une absorption intestinale anormalement élevée des stérols alimentaires, probablement couplée à leur défaut d'excrétion hépatique, et dont la conséquence majeure est l'accumulation de stérols athérogènes.

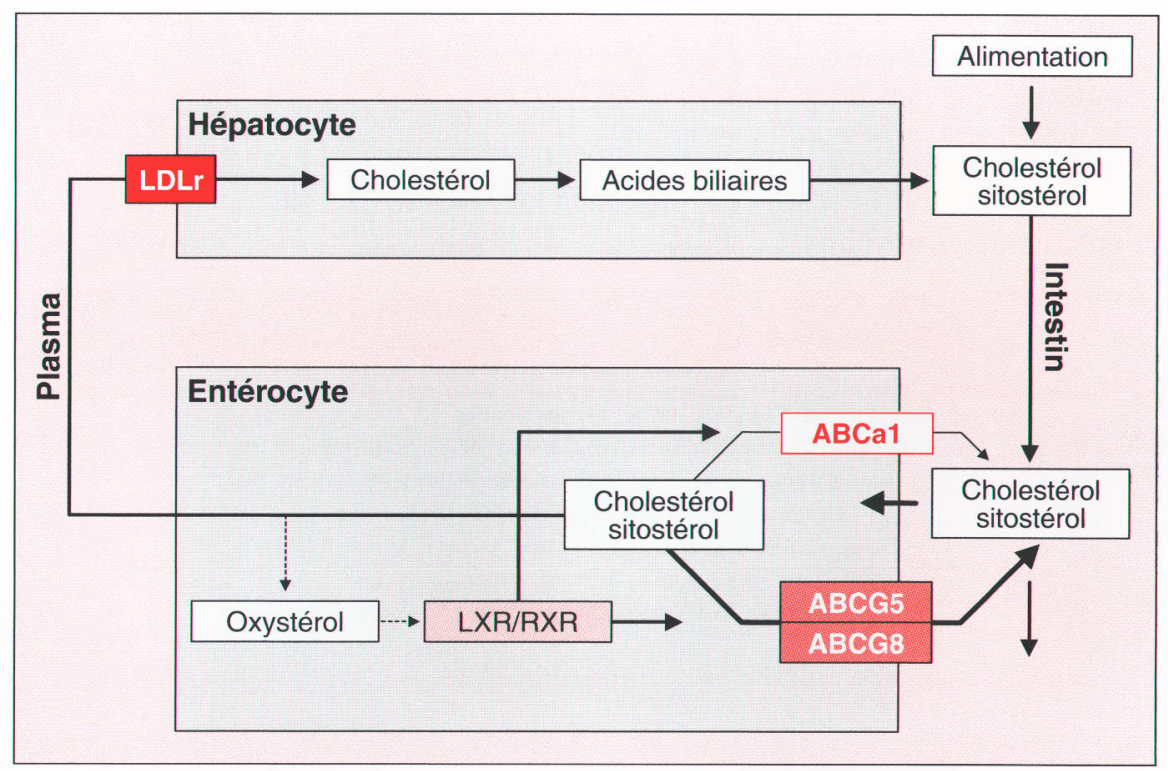

Figure 1. Régulation de la sécrétion hépatique et de l'absorption intestinale des stérols. Sécrétés dans l'intestin les acides biliaires permettent la solubilisation des stérols alimentaires (cholestérol et sitostérol), facilitant ainsi leur absorption par les entérocytes. L'augmentation de la teneur en stérols dans l'entérocyte provoque l'activation de RXR/LXR, induisant à son tour l'expression des transporteurs ABCA1, ABCG5 et ABCG8. Ces transporteurs permettent la sécrétion d'une petite partie du cholestérol absorbé et de la majorité des stérols d'origine végétale (sitostérol) vers la lumière intestinale. Chez les patients sitostérolémiques, cette dernière voie métabolique est défectueuse et les stérols d'origine végétale s'accumulent dans le plasma et les organes périphériques. 


\section{Q uand l'endocrinologie inverse rejoint le clonage positionnel}

Comme cela a été décrit récemment, l'absorption digestive du cholestérol est contrôlée par les hétérodimères que forment les récepteurs nucléaires liver $X$ receptor ( $L X R)$ et retinoid $X$ receptor ( $R X R$ ) qui augmentent l'expression de ABCAl dans les entérocytes ([3] et $\mathrm{m} / \mathrm{s} 2000, n^{\circ} 12$, p. 1456) (figure 1). C'est à partir de cette observation que Berge et al. ont émis l'hypothèse que le gène défecteux chez les patients atteints de sitostérolémie pourrait également être contrôlé par LXR/RXR, et coder pour une protéine dont le rôle serait de limiter l'absorption intestinale du cholestérol et surtout des sistostérols. L'utilisation de microarrays leur a permis d'identifier un EST (expressed sequence tag) dont I'expression est augmentée dans l'intestin et le foie de souris traitées par un agoniste de LXR capable de moduler l'absorption des stérols alimentaires [4]. La séquence obtenue présentait de fortes similitudes avec celles des transporteurs White de la drosophile, un sous-groupe de la famille des gènes à ATP binding cassette. Le clonage de l'ADNC puis du gène correspondant, nommé $A B C G 5$, leur a permis d'identifier une mutation introduisant un codon stop chez un de leurs patients atteints de sitostérolémie. Cependant, aucun des huit autres patients étudiés ne présentait $d$ 'anomalie du gène $A B C G 5$. En criblant les différentes banques génomiques disponibles, Berge et al. identifièrent un autre gène de la famille White nommé $A B C G 8$ et localisé à proximité de $A B C G 5$. Leurs huit patients présentaient tous une mutation de ce gène, quatre à l'état homozygote et quatre à l'état hétérozygote. Enfin, leur étude montre que $A B C G 5$ et $A B C G 8$ sont exprimés uniquement dans le foie et l'intestin et que leur expression augmente si les souris sont soumises à un régime alimentaire riche en cholestérol [4].

Simultanément à ces travaux, une autre équipe a, quant à elle, utilisé une stratégie de clonage positionnel [5]. Elle avait précédemment localisé le gène défectueux sur le chromo- timorgans [6], et révèle maintenant qu'elle contient trois EST exprimés dans le foie et l'intestin, dont l'un correspond au gène $A B C G 5$ [5]. Parmi les trente patients atteints de sitostérolémie, neuf présentent des mutations de ce gène et, comme dans l'étude précédente, le foie et I'intestin sont les sites physiologiques d'expression d'ABCG5. A la lumière des résultats de Berge et al., Lee et al. ont ensuite montré que leurs patients présentaient tous une mutation soit pour $A B C G 5$, soit pour ABCG8 [7] Les protéines White sont ce que l'on appelle des semi-transporteurs car elles ont la particularité de ne posséder que six segments transmembranaires, et non pas douze comme les protéines $A B C$ classiques dont $A B C A 1$, et de n'être fonctionnelles que sous forme d'hétérodimères. Les protéines ABCG 5 et ABCG 8 font partie de ce groupe de semi-transporteurs. Leurs gènes, qui sont localisés, au même locus, en tandem mais têtebêche, ont un promoteur bidirectionnel et des éléments de contrôle communs [8]. Même si le mécanisme moléculaire exact de fonctionnement de ces deux semi-transporteurs n'est pas encore connu, on peut envisager qu'ils fonctionnent aussi en tandem pour former un transporteur fonctionnel dont le déficit se traduit par un défaut d'exclusion des stérols autres que le cholestérol et non par un défaut d'absorption entérocytaire (figure 1).

\section{Rôle intégré des transporteurs $A B C$ dans le métabolisme du cholestérol}

Ainsi, deux maladies métaboliques, la maladie de Tangier et la sitostérolémie, sont à l'origine de la compréhension du rôle de trois transporteurs de la famille $A B C$ et de leur contrôle, notamment par RXR/ LXR. On peut souligner que l'expression d'un autre transporteur de la même famille, $A B C G 1$, qui semble impliqué dans la régulation de l'homéostasie du cholestérol intracellulaire dans les macrophages [9], est également contrôlée par RXR/ LXR. La compréhension des mécanismes de contrôle de ces gènes par les récepteurs nucléaires RXR/LXR constitue un champ d'investigation prometteur dans la lutte contre I'athérosclérose. Prochaine étape, l'obtention de souris génétiquement modifiées devrait permettre d'évaluer l'importance et le rôle de ces transporteurs dans I'homéostasie des stérols au niveau intestinal et hépatique.

1. Wade DP, O wen JS. Regulation of the cholesterol efflux gene, ABCA1. Lancet 2001; 357: 161-3. 2. Bjorkhem I, Muri Boberg K, Leitersdorf E. Inborn Errors in bile acid biosynthesis and storage of sterols other than cholesterol. In The M etabolic Bases of Inherited Diseases 2961-2988 (McGraw Hill, N ew York, 2001).

3. Repa JJ, Turley SD, Laboccaro JA, et al. Regulation of absorption and ABC1-mediated efflux of cholesterol by RXR heterodimers. Science 2000; 289: 1524-9.

4. Berge KE, Tian H, Graf GA, et al. Accumulation of dietary cholesterol in sitosterolemia caused by mutations in adjacent ABC transporters. Science 2000; 290: 1771-5.

5. Lee MH, Lu K, Hazard S, Yu HW, et al. Identification of a gene, $A B C G 5$, important in the regulation of dietary cholesterol absorption. $\mathrm{N}$ at $\mathrm{Genet}$ 2001; 27: 79-83

6. Patel SB, Salen G, Hidaka H, et al. Mapping a gene involved in regulating dietary cholesterol absorption. The sitosterolemia locus is found at chromosome 2p21. J Clin Invest 1998; 102: 10414.

7. Lee MH, Lu K, Patel SB. Genetic basis of sitosterolemia. Curr Opin Lipidol 2001:12: 141-9.

8. Allayee $H$, Laffitte BA, Lusis AJ. Biochemistry An absorbing study of cholesterol. Science 2000; 290: 1709-11.

9. Klucken J, Buchler C, Orso E, et al. ABCG1 ( $A B C 8)$, the human homolog of the Drosophila white gene, is a regulator of macrophage cholesterol and phospholipid transport. Proc Natl Acad Sci USA 2000; 97 : 817-22.

\section{Gilles Lambert}

Molecular Disease Branch, National Heart Lung and Blood Institute, National Institutes of $H$ ealth, Bethesda, MD 20890, États-Unis.

\section{Michel Krempf}

Clinique d'endocrinologie, H ôtel-Dieu, 44093 Nantes Cedex 01, France. email : mkrempf@sante.univ-nantes.fr 